\title{
寄生原生动物线粒体与适应性进化
}

\author{
田海峰 ${ }^{1,2}$, 文建凡 ${ }^{1, *}$ \\ (1. 中国科学院昆明动物研究所 遗传资源与进化国家重点实验室, 云南 昆明 $650223 ; 2$. 中国科学院研究生院, 北京 100049)
}

摘要: 真核生物的线粒体一般具有一定的典型的结构和功能。然而, 在单细胞的寄生原生动物中却不断发 现从数量、结构到功能均与典型线粒体明显不同的线粒体, 表现出线粒体的巨大可塑性和丰富的多样性。该文 对寄生原生动物中这些多样的线粒体进行了概述, 并对形成这种多样性的根本原因, 即这些生物对寄生生活微 氧或无氧环境线粒体所发生的种种适应性进化进行了分析探讨。

关键词: 寄生原生动物; 线粒体; 多样性; 适应性进化

中图分类号: Q349; Q959.113.8 文献标志码: A 文章编号: 0254-5853-(2010)01-0035-04

\section{Diversity of Parasitic Protozoan Mitochondria and Adaptive Evolution}

\author{
TIAN Hai-Feng ${ }^{1,2}$, WEN Jian-Fan ${ }^{1, *}$ \\ (1. State Key Laboratory of Genetic Resources and Evolution, Kunming Institute of Zoology, the Chinese Academy of Sciences, Kunming \\ 650223, China; 2. Graduate School of the Chinese Academy of Sciences, Beijing 100049, China)
}

\begin{abstract}
Eukaryotic mitochondrion generally possess a definite and canonical structure and function. However, in the unicellular parasitic protozoa, various atypical mitochondria with respect to the number, structure, and function, have been discovered consecutively, revealing the variability, plasticity and rich diversity of mitochondrion. Here, we review the mitochondrial diversity in diverse parasitic protozoa, and the underlying reason for such diversity — the adaptive evolution of mitochondrion to the micro-oxygen or anaero parasitic environment of these parasites is also analyzed and discussed.
\end{abstract}

Key words: Parasitic protozoa; Mitochondria; Diversity; Adaptive evolution

线粒体是真核细胞中普遍存在的重要细胞器 之一。典型的线粒体由内、外双层膜包被的棒状 结构, 双层膜之间有膜间隙, 内膜内陷成许多 嵴, 内膜以内为基质, 基质内含有线粒体自身的 基因组。线粒体的两个最重要的功能途径一三 羧酸循环(TCA)和氧化磷酸化就分别发生在基质中 和内膜上。通过这两个功能途径产生大量的 ATP, 为细胞提供能量, 因而线粒体有 “细胞的动力工厂” 之称。此外, 线粒体还具有许多其他功能, 如参 与细胞活动的调节及多种生物大分子的合成等 （Ashkenazi \& Dixit, 1998; Giegé et al, 2008）。然
而, 作为单细胞真核生物的原生动物, 它们中许 多种类的线粒体无论是结构还是功能方面却发生 了很大程度“特化”或“退化”, 如动基体类的线粒体 “巨型化”为动基体 (kinetoplastid), 而另一些种类 (如阿米巴类、双滴虫类和厌氧纤毛虫类等) 中, 甚至变得与典型线粒体的差异如此之大 [如产氢体

(hydrogensome) 和 mitosome], 以致于人们曾一度 认为这些生物不具有线粒体。本文拟对原生动物 中所发现线粒体的结构与功能多样性作一概述, 并对这些多样性形成的进化生物学意义进行探 讨。

收稿日期: 2009-12-07; 接受日期：2009-12-21

基金项目：国家基金委创新群体项目（30021004）; 云南省基础研究计划项目（2006C0014Z，2007C098M）; 中科院重要方向项目 (KSCX2-YW-R-091)

*通讯作者（Corresponding author）, E-mail: wenjf@mail.kiz.ac.cn 第一作者简介: 田海峰, 男, 博士研究生, 主要从事原生动物与代谢途径的进化 


\section{1 原生动物中多样的 “线粒体”}

\section{1 具有最小基因组的线粒体}

顶复门类 (Apicomplexa) 的细胞中线粒体只有 一个, 其内部结构也与典型线粒体不同, 如症原虫 线粒体的嵴在生活史的不同阶段发生不同的变化

（Krungkrai et al, 1999）。更重要的是这类生物的 线粒体基因组是目前已知的最小线粒体基因组, 仅约 6 7 kb, 如疮原虫的线粒体基因组仅编码电 子传递链 (ETC) 的 3 个蛋白 (细胞色素 $c$ 氧化酶 亚基 I、II, 以及脱血红素细胞色素 $c$ ）和一些核糖 体 RNA（Vaidya et al, 1989），而且这些编码基因 在不同物种线粒体基因组的排布也不同。顶复体 类多数物种均具有 ETC 复合物的大多数成份及参 与 TCA 循环的酶（Seeber et al, 2008）。疮原虫在 血液阶段的 ETC 各复合物的酶活性、氧的消耗以 及参与 TCA 循环的各酶表达均较低 (Llinás et al, 2006; Painter et al, 2007), 但与呼吸及 TCA 循环 相关基因的表达在感染宿主的不同阶段却有着明 显不同 (Daily et al, 2007; Lovegrove et al, 2008), 提示症原虫的线粒体可能在不同的阶段功能发生 着变化。有趣的是, 症原虫丙酮酸脱氢酶 $(\mathrm{PDH})$ 定位在顶复体一一另一细胞器中, 而非定位于线 粒体中（Foth et al, 2005）。

\section{2 巨型化的线粒体一一动基体}

典型线粒体相对细胞来说是很小的, 然而动 基体类 (kinetoplastids) 生物尽管只有一个线粒体, 但体积却变得很大, 在特定条件下可占据细胞体 积的 13\%（de Souza et al, 2009）, 因而这种线粒体 被专门称为动基体, 这类生物也因此而得名。动 基体的基因组占到细胞总 DNA 量的 30\%, 由两种 相互连结的大小环组成, 称为 $\mathrm{kDNA}$, 其中大环编 码线粒体蛋白及线粒体 rRNAs; 小环则编码引导 RNA 参与对大环转录基因的编辑(Yatawara et al, 2008)。动基体随外界营养条件的改变而变化, 在 葡萄糖丰富的环境中变得很小, 与另一细胞器

一酵解体成此消彼长的变化 (Faria-e-Silva et al, 2000）。动基体的内部结构也随生活周期的变化而 变化, 如雉虫动基体在动物宿主阶段不具嵴状结 构, 而在昆虫消化道内则具有层状峳。雉虫在哺 乳动物宿主阶段主要依赖酵解体提供能量, 而动 基体处于抑制状态（Tielens et al, 1998），在昆虫消 化道阶段动基体则通过 TCA 循环把丙酩酸和部分
氨基酸 (如脯氨酸, 苏氨酸) 进一步降解, 并具有 一条与植物 ETC 类似的呼吸链 (van Weelden et al, 2005）。

\section{3 能产氢的 “线粒体” 一一产氢体}

产氢体(hydrogensome) 是一种双层膜包被、可 产ATP及 $\mathrm{H}_{2}$ 的细胞器。该细胞器首先发现于毛滴虫 （Lindmark，1973），后又从多种厌氧纤毛虫和 Blastocystis等物种中鉴定出来 (Akhmanova et al, 1998; Nasirudeen et al, 2004; Yarlett et al, 1981)。 在结构上, 毛滴虫产氢体由双层薄而平滑的膜包 被，双层膜无间隙存在（Benchimol，2009），但 Nyctotherus及Blastocystis产氢体中有着明显的嵴状 结构（Dunn et al, 1989; Zierdt et al, 1988）。很多 物种, 如毛滴虫 (Clemens \& Jhonson, 2000) 、瘤胃 纤毛虫的产氢体不具有基因组, 但最近在 Nyctotherus及Blastocystis的产氢体中却发现了基因 组的存在 (Akhmanova et al, 1998; Wawrzyniak et al, 2008）。在功能上, 毛滴虫产氢体上的研究表明: 通过丙酮酸: 铁氧还蛋白氧化还原酶（PFO）降解 丙酮酸为乙酰辅酶 $\mathrm{A}$, 并最终生成 $\mathrm{H}_{2}$ 、乙酸和 $\mathrm{H}_{2} \mathrm{O}$, 并通过底物水平磷酸化生成ATP, 还负责 $\mathrm{FeS}$ 簇的合成与组装（Sutak et al, 2004）及部分氨基 酸代谢 (Hrdý et al, 2007)。与毛滴虫产氢体相比, 纤毛虫Nyctotherus及Blastocystis的产氢体在功能上 更为复杂，不仅产氢，还有着很多线粒体的其他 功能 (Hackstein et al, 2007; Stechmann et al, 2008）。有意思的是, 瘤胃纤毛虫Dasytricha产氢 体则与毛滴虫产氢体类似, 并不具有参与ETC及 TCA的相关酶（Hackstein et al, 2007），这反应了不 同纤毛虫的产氢体在功能上的差异很大。

\section{4 极端特化的 “线粒体” — mitosome}

近些年，在一些既无线粒体又产氢体的原生 动物中 (如阿米巴、贾第虫、脑炎微狍子虫、隐狍 子虫) 发现了一类双层膜包被的细胞器（Mai et al, 1999; Riordan et al, 1999; Tovar et al,2003; Williams et al, 2002), 称之为 mitosome。贾第虫及 隐孢子虫的 mitosome 的内外膜具有间隙, 但阿米 巴及微孢子虫的 mitsome 中则不存在 ( Tachezy et al, 2007)。Mitosome 不具有自身基因组 (León-Avila et al, 2004）。在功能上, mitosome 均不具有 TCA 循 环及氧化磷酸化等功能, 其已经实验确认的功能 仅为 $\mathrm{FeS}$ 笶合成 (Goldberg et al, 2008; LaGier et al, 2003; Tovar et al, 2003)。然而, 对发现 mitosome 
的物种的核基因组分析表明, mitosome 可能也具 有一些由核基因编码的其他功能的蛋白, 如 Encephalitozoon cuniculi 中具有丙酮酸脱氢酶复合 物 $\mathrm{E} 1$ 的 $\alpha 、 \beta$ 亚基（Katinka et al, 2001）、甘油 3 磷酸脱氢酶和含锰超氧化物岐化酶等 (Burri et al, 2006），但这些功能并未得到实验验证。我们最近 对 Giardia lamblia 基因组的分析也显示该物种完全 不具有参与 TCA 循环及氧化磷酸化的各个酶的同 源物, 但其 mitosome 仍可能有着参与磷脂合成或 脂肪酸氧化代谢的功能（Tian，2008）。

\section{2 多样性形成的根本原因一一对寄生生活 的适应性进化}

为什么这些寄生原生动物中的“线粒体”会发生 如此这般的变化而形成了这些多样化的线粒体。

寄生原生动物作为单细胞的寄生虫, 寄生于 宿主体内, 甚至细胞内, 这样的生活环境的一个重 要的特点就是它们接触不到游离的氧分子, 即它 们生活在一个微氧或无氧的环境。而线粒体作为 细胞内利用氧分子进行氧化呼吸生产 ATP 的胞 器, 在这种微氧或无氧的条件下势必首当其冲地 面临着选择压力。这些生物正是在这种共同选择 压力的长期作用下, 又由于各自的寄生环境有所 不同，它们中的线粒体发生了既相似又有所不同 的适应性进化。相似的方面体现在在大的进化策 略上有着相同的进化趋势, 即针对氧的缺乏, 线 粒体中的三羧酸循环和氧化呼吸链发生着改组、 退化或丢失, 降低氧的消耗, 同时也就降低或失 去 ATP 的生产能力。不同的方面则体现在由于寄 生环境的有所不同，线粒体发生不同程度的“退化” 或“特化”, 如同属纤毛虫类的瘤胃纤毛虫与 Nyctotherus 均具有产氢体, 但它们生活于不同的

\section{参考文献:}

Akhmanova A, Voncken F, van Alen T, van Hoek A, Boxma B, Vogels G, Veenhuis M, Hackstein JH. 1998. A hydrogenosome with a genome[J]. Nature, 396(6711): 527-528.

Ashkenazi A, Dixit VM. 1998. Death receptors: signaling and modulation[J]. Science, 281(5381): 1305-1308.

Benchimol M. 2009. Hydrogenosomes under microscopy[J]. Tissue Cell, 41(3): 151-168.

Burri L, Williams BA, Bursac D, Lithgow T, Keeling PJ. 2006. Microsporidian mitosomes retain elements of the general mitochondrial targeting system[J]. Proc Natl Acad Sci USA, 103: 15916-15920.

Clemens DL, Johnson PJ. 2000. Failure to detect DNA in hydrogenosomes of
环境中，其产氢体在结构及功能上有所不同；再 如顶复体和动基体类的线粒体在不同的生活史阶 段也有着不同的形态与功能, 在营养物质有限的 情况下会增大体积以弥补数量的不足。这提示线 粒体结构与功能与所生活环境的营养条件密切相 关。

正是由于这些原因, 寄生原生动物中的“线粒 体”产生了丰富的多样性。

\section{3 展 望}

寄生原生动物“线粒体”的多样性将会随着对更 多原生动物的研究而有更多的发现, 如部分寄生 厌氧纤毛虫可能也具有 mitosome (Hackstein et al, 2007），但有待证实。这些发现必将增进人们对线 粒体可塑性及线粒体适应性进化的更深入认识。

人们对上述寄生原生动物“线粒体”的功能已有 所认识, 但还远不够深入、全面, 如顶复体类、动 基体类生物的线粒体在不同生活史阶段的功能及 其差异仍有待进一步研究; 再如, 具产氢体的物种 核基因组的注释显示不同物种的产氢体可能在功 能上有着较大差异，这些不同的功能及其成因都 有待验证及认识。另外, 已发现的 mitosome 的功 能仅为参与 $\mathrm{FeS}$ 蛋白簇合成, 利用各种手段来认识 该胞器的组份、功能及其起源是当前对 mitosome 研究的热点。

对这些不同的“线粒体”进行比较, 将有助于理 解这些细胞器在功能上是如何进化的, 将进一步 有助于理解寄生原生动物“线粒体”是如何在形态结 构及代谢途径上与不同的宿主环境相适应的，这 也有助于理解这些致病原生动物是如何进化发展 的，并从而有可能从适应性进化的角度去寻找和 发现有效的靶标用于这些寄生原生动物的防治。
Trichomonas vaginalis by nick translation and immunomicroscopy[J] Mol Biochem Parasitol, 106(2): 307-313.

Daily JP, Scanfeld D, Pochet N, Le Roch K, Plouffe D, Kamal M, Sarr O, Mboup S, Ndir O, Wypij D, Levasseur K, Thomas E, Tamayo P, Dong C, Zhou Y, Lander ES, Ndiaye D, Wirth D, Winzeler EA, Mesirov JP, Regev A. 2007. Distinct physiological states of Plasmodium falciparum in malaria-infected patients[J]. Nature, 450(7172): 1091-1095.

de Souza W, Attias M, Rodrigues JC. 2009. Particularities of mitochondrial structure in parasitic protists (Apicomplexa and Kinetoplastida)[J]. Int $J$ Biochem Cell Biol, 41(10): 2069-2080.

Dunn LA, Boreham PF, Stenzel DJ. 1989. Ultrastructural variation of 
Blastocystis hominis stocks in culture[J]. Int J Parasitol,19(1): 43-56.

Faria-e-Silva PM, Attias M, de Souza W. 2000. Biochemical and ultrastructural changes in Herpetomonas roitmani related to the energy metabolism[J]. Biol Cell, 92(1): 39-47.

Foth BJ, Stimmler LM, Handman E, Crabb BS, Hodder AN, McFadden GI. 2005. The malaria parasite Plasmodium falciparum has only one pyruvate dehydrogenase complex, which is located in the apicoplast[J]. Mol Microbiol, 55(1): 39-53.

Giegé P, Grienenberger JM, Bonnard G. 2008. Cytochrome c biogenesis in mitochondria[J]. Mitochondrion, 8(1): 61-73.

Goldberg AV, Molik S, Tsaousis AD, Neumann K, Kuhnke G, Delbac F, Vivares CP, Hirt RP, Lill R, Embley TM. 2008. Localization and functionality of microsporidian iron-sulphur cluster assembly proteins[J]. Nature, 452(7187): 624-628.

Hackstein JHP, de Graaf RM, van Hellemond JJ, Tielens AGM. 2007. Hydrogenosomes of Anaerobic Ciliates [M]// Alexander $\mathrm{S}$ ed. Microbiology Monographs: Vol. 9: Jan T ed .Hydrogenosomes and Mitosomes: Mitochondria of Anaerobic Eukaryotes. Berlin/Heidelberg:Springer-Verlag, 97-112.

Hrdý I. 2007. Metabolism of Trichomonad Hydrogenosomes[M]// Alexander S ed. Microbiology Monographs :Vol. 9 Jan T ed .Hydrogenosomes and Mitosomes: Mitochondria of Anaerobic Eukaryotes. Berlin/Heidelberg:: Springer-Verlag 113-145.

Katinka MD, Duprat S, Cornillot E, Méténier G, Thomarat F, Prensier G, Barbe V, Peyretaillade E, Brottier P, Wincker P, Delbac F, El Alaoui H, Peyret P, Saurin W, Gouy M, Weissenbach J, Vivarès CP. 2001. Genome sequence and gene compaction of the eukaryote parasite Encephalitozoon cuniculi[J]. Nature, 414(6862): 450-453.

Krungkrai S, Leangaramgul P, Kudan S, Prapunwattana P, Krungkrai J. 1999. Mitochondrial heterogeneity in Human malarial parasite Plasmodium falciparum[J]. ScienceAsia, 25: 77-83.

LaGier MJ, Tachezy J, Stejskal F, Kutisova K, Keithly JS. 2003. Mitochondrial-type iron-sulfur cluster biosynthesis genes (IscS and $\mathrm{IscU})$ in the apicomplexan Cryptosporidium parvum[J]. Microbiology, 149(Pt 12): 3519-3530.

León-Avila G, Tovar J. 2004. Mitosomes of Entamoeba histolytica are abundant mitochondrion-related remnant organelles that lack a detectable organellar genome[J]. Microbiology, 150(Pt 5): 1245-1250.

Lindmark DG, Müller M. 1973. Hydrogenosome, a cytoplasmic organelle of the anaerobic flagellate Tritrichomonas foetus, and its role in pyruvate metabolism[J]. J Biol Chem, 248(22): 7724-7728.

Llinás M, Bozdech Z, Wong ED, Adai AT, DeRisi JL. 2006. Comparative whole genome transcriptome analysis of three Plasmodium falciparum strains[J]. Nucleic Acids Res, 34(4): 1166-1173.

Lovegrove FE, Peña-Castillo L, Liles WC, Hughes TR, Kain KC. 2008. Plasmodium falciparum shows transcriptional versatility within the human host[J]. Trends Parasitol, 24(7): 288-291.

Mai Z, Ghosh S, Frisardi M, Rosenthal B, Rogers R, Samuelson J. 1999. Hsp60 is targeted to a cryptic mitochondrion-derived organelle ("crypton") in the microaerophilic protozoan parasite Entamoeba histolytica[J]. Mol Cell Biol, 19(3): 2198-2205.

Nasirudeen AM, Tan KS. 2004. Isolation and characterization of the mitochondrion-like organelle from Blastocystis hominis[J]. J Microbiol Methods, 58(1): 101-109.

Painter HJ, Morrisey JM, Mather MW, Vaidya AB. 2007. Specific role of mitochondrial electron transport in blood-stage Plasmodium falciparum[J]. Nature, 446(7131): 88-91.
Riordan CE, Langreth SG, Sanchez LB, Kayser O, Keithly JS. 1999. Preliminary evidence for a mitochondrion in Cryptosporidium parvum: phylogenetic and therapeutic implications[J]. J Eukaryot Microbiol, 46: 52S-55S.

Seeber F, Limenitakis J, Soldati-Favre D. 2008. Apicomplexan mitochondrial metabolism: a story of gains, losses and retentions[J]. Trends Parasitol, 24(10): 468-478.

Stechmann A, Hamblin K, Pérez-Brocal V, Gaston D, Richmond GS, van der Giezen M, Clark CG, Roger AJ. 2008. Organelles in Blastocystis that blur the distinction between mitochondria and hydrogenosomes[J]. Curr Biol, 18(8):580-585.

Sutak R, Dolezal P, Fiumera HL, Hrdy I, Dancis A, Delgadillo-Correa M, Johnson PJ, Müller M, Tachezy J. 2004. Mitochondrial-type assembly of FeS centers in the hydrogenosomes of the amitochondriate eukaryote Trichomonas vaginalis[J]. Proc Natl Acad Sci USA, 101(28): 10368-10373.

Tachezy J, Smid O. 2007. Mitosomes in Parasitic Protists [M]// Alexander S ed. Microbiology Monographs : Vol. 9 Jan T ed .Hydrogenosomes and Mitosomes: Mitochondria of Anaerobic Eukaryotes. Berlin/Heidelberg:Springer-Verlag, 201-230.

Tian HF. 2008. Identification of an incomplete glycerophopholipid biosynthesis pathway in Giardia lamblia and the evolution of glycerophopholipid biosynthesis pathway in Eukaryotes[D]. Kunming Institute of Zoology, the Chinese Academy of Sciences. [田海峰. 2008. 贾第虫不完整甘油磷脂合成途径的鉴定及甘油磷脂合成途径在真 核生物中的进化. 中国科学院昆明动物研究所博士论文.]

Tielens AG, Van Hellemond JJ. 1998. Differences in energy metabolism between Trypanosomatidae[J]. Parasitol Today, 14(7): 265-272.

Tovar J, León-Avila G, Sánchez LB, Sutak R, Tachezy J, van der Giezen M, Hernández M, Müller M, Lucocq JM. 2003. Mitochondrial remnant organelles of Giardia function in iron-sulphur protein maturation[J]. Nature, 426(6963): 172-176.

van Weelden SW, van Hellemond JJ, Opperdoes FR, Tielens AG. 2005. New functions for parts of the Krebs cycle in procyclic Trypanosoma brucei, a cycle not operating as a cycle[J]. J Biol Chem, 280(13): 12451-12460.

Vaidya, AB, Akella R, Suplick K. 1989. Sequences similar to genes for two mitochondrial proteins and portions of ribosomal RNA in tandemly arrayed 6-kilobase-pair DNA of a malarial parasite[J]. Mol Biochem Parasitol, 35(2): 97-107.

Wawrzyniak I, Roussel M, Diogon M, Couloux A, Texier C, Tan KS, Vivarès CP, Delbac F, Wincker P, El Alaoui H. 2008. Complete circular DNA in the mitochondria-like organelles of Blastocystis hominis[J]. Int $J$ Parasitol, 38(12): 1377-1382.

Williams BAP, Hirt RP, Lucocq JM, Embley TM. 2002. A mitochondrial remnant in the microsporidian Trachipleistophora hominis[J]. Nature, 418(6900): 865-869.

Yarlett N, Hann AC, Lloyd D, Williams A. 1981. Hydrogenosomes in the rumen protozoon Dasytricha ruminantium Schuberg[J]. Biochem J.,200(2):365-372.

Yatawara L, Le TH, Wickramasinghe S, Agatsuma T. 2008. Maxicircle (mitochondrial) genome sequence (partial) of Leishmania major: gene content, arrangement and composition compared with Leishmania tarentolae[J]. Gene, 424(1-2): 80-86.

Zierdt CH, Donnolley CT, Muller J, Constantopoulos G. 1988. Biochemical and ultrastructural study of Blastocystis hominis[J]. J Clin Microbiol, 26(5): 965-970. 